\title{
Electron probe microanalysis of transition metals using L-lines: the effect of self- absorption
}

Xavier Llovet ${ }^{1}$, Aurélien Moy ${ }^{2}$ and John Fournelle ${ }^{2}$

${ }^{1}$ University of Barcelona, United States, ${ }^{2}$ Department of Geoscience, University of Wisconsin-Madison, United States

The use of soft X-rays $(1<\mathrm{keV})$ for electron probe microanalysis (EPMA) poses numerous difficulties mainly because soft X-rays involve valence electrons, which are affected by chemical bonding, and matrix corrections do not account for bonding effects [1]. Because of that, alternative strategies are being developed [2]. In the case of the main L-lines of the first-row transition metals, the situation is further complicated by the effect of self-absorption. This is so because these lines are located close to the $\mathrm{L}_{2}$ and $\mathrm{L}_{3}$ absorption edges and the high-energy side of the lines straddle the rising L-edges. As a result, there is a distortion of the shape and position of the lines with incident electron energy. Although the effect of self-absorption has been known for decades (mainly from soft X-ray spectroscopic studies of solids [3]), there is still little understanding of its implications in quantitative EPMA analysis. In this study, we assess the influence of self-absorption on the L $\alpha$ lines emitted from metallic $\mathrm{Fe}, \mathrm{Ni}, \mathrm{Cu}$ and $\mathrm{Zn}$ targets. X-ray emission lines are modelled as Lorentzian distributions, with intensities obtained from Monte Carlo simulations [4], and self-absorption is evaluated numerically by using experimental energy-dependent mass absorption coefficients, $(\mu / \rho)(E)$, where $E$ is the photon energy, and X-ray depth-distributions obtained from Monte Carlo simulations. For comparison purposes, self-absorption is also evaluated using a definite mass absorption coefficient, $(\mu / \rho)$, which is the conventional approach used in matrix corrections.

Figure 1a shows the theoretical Ni L $\alpha$ emission line (a Lorentzian profile with a natural width of $2.58 \mathrm{eV}$ [5]), and the energy-dependent mass absorption coefficients of metallic Ni measured by Ménesguen et al. [6]. Both the emission line and the absorption edge are relatively broad and overlap is considerable; as a result, $(\mu / \rho)(E)$ increases rapidly across the X-ray emission line. Figure $1 \mathrm{~b}$ compares the emitted Ni L $\alpha$ line for $30 \mathrm{keV}$ electron excitation evaluated using the mass absorption coefficients of Ménesguen et al., with that obtained using a fixed mass absorption coefficient of $(\mu / \rho)=3772 \mathrm{~cm}^{2} / \mathrm{g}$, i.e., the value measured by the same authors at the central energy of the $\mathrm{Ni} L \alpha$ line $(E=851.47 \mathrm{eV})$. There is a considerable distortion of the line shape when self-absorption is calculated using $(\mu / \rho)(E)$, which results in a shifting of the line position towards lower energy and a $\sim 15 \%$ increase of its peak height. This suggests that, in this case, even if we know accurately the mass absorption coefficient at the line energy (e.g., from firstprinciple calculations [7] or experimental measurements [6]), errors of up $\sim 15 \%$ can still be made if selfabsorption is not accounted for. The observed intensity difference decreases with decreasing incident electron energy and becomes negligible below $\sim 5 \mathrm{keV}$ (Fig. 1c). This trend is confirmed when both the diagram and satellite lines are included in the calculations and the resulting spectrum is convolved with a Gaussian distribution to account for spectrometer broadening. Because the structure and position of the rising $\mathrm{L}_{2,3}$-edge depends on the chemical bonding, for $\mathrm{Ni}$ compounds the degree of self-absorption will vary depending on the compound, leading to errors in the evaluated concentrations.

Calculations for metallic Fe show similar results than those for metallic Ni. In contrast, self-absorbed Xray intensities evaluated for metallic $\mathrm{Zn}$ and $\mathrm{Cu}$ using $(\mu / \rho)(E)$ do not differ significantly from those obtained using a single $(\mu / \rho)$ value. This is so because for metallic $\mathrm{Zn}$ and $\mathrm{Cu}$, there is almost no overlap between the emission $\mathrm{L} \alpha, \beta$-line and the absorption $\mathrm{L}_{2,3}$-edge. However, overlap may exist for some $\mathrm{Cu}$ and $\mathrm{Zn}$ compounds [8]. 


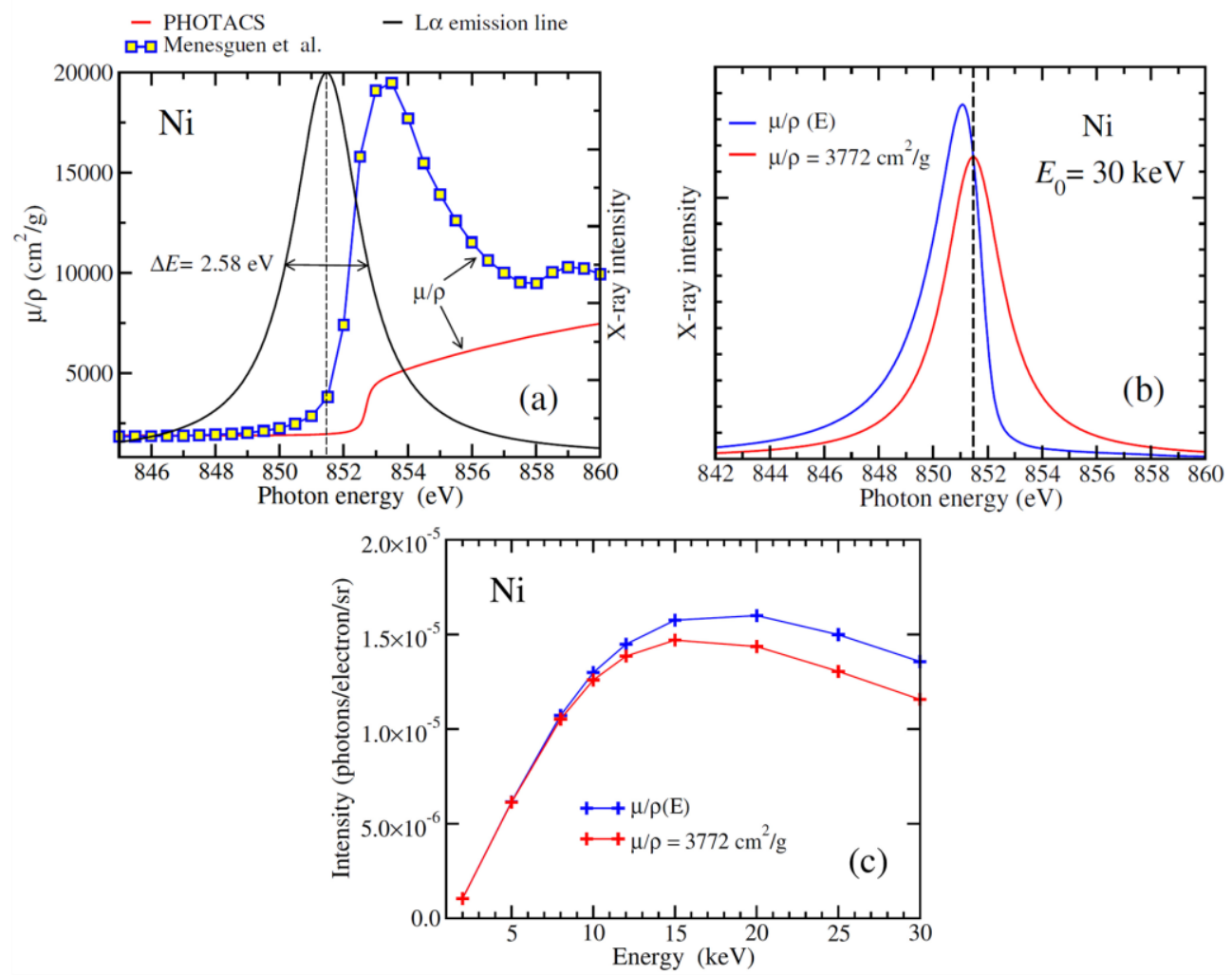

Figure 1. Ni L $\alpha$ emission line and theoretical (PHOTACS [7]) and experimental (Ménesguen et al. [6]) mass absorption coefficients of $\mathrm{Ni}$ (a). Comparison of self-absorbed $\mathrm{Ni} \mathrm{L} \alpha$ emission lines for $30 \mathrm{keV}$ electron excitation (b) and X-ray intensities as a function of incident electron energy (c) calculated with the energy-dependent mass absorption coefficients of Ménesguen et al. $(\mu / \rho(E))$ and with $(\mu / \rho)=3772$ $\mathrm{g} / \mathrm{cm}^{2}$.

\section{References}

[1] X Llovet et al., Microsc. Microanal. 22 (2016), p. 1233.

[2] A Moy et al., Microsc. Microanal. 25 (2019), p. 664.

[3] RJ Liefeld in "Soft X-ray band spectra" (1968), ed. DJ Fabian (Academic Press, London), p. 133.

[4] X Llovet and F Salvat, Microsc. Microanal. 23 (2017), p. 634.

[5] C Bonnelle, Ann Phys 1 (1966), p. 439.

[6] Y Ménesguen et al., Metrologia 55 (2018), p. 56.

[7] L Sabatucci and F Salvat, Rad. Phys. Chem. 121 (2016), p. 122.

[8] We thank Y. Ménesguen and M.C Lepy for providing the numerical data from Ref. [6]. 